\title{
Antikes in der Grabrede des Georgios Akropolites anf Johannes Dukas.
}

Die byzantinischen Königsspiegel, Lob- und Grabreden auf Fürsten und andere für Staat oder Kirche bedeutsame Persönlichkeiten lassen in recht greifbarer Weise einen Zug hervortreten, der die byzantinische Literatur überhaupt für den Literaturhistoriker besonders interessant und reich an reizvollen Problemen gestaltet. Die Mannigfaltigkeit der Voraussetzungen des geistigen Lebens in Byzanz, die Verschiedenartigkeit der dort sich kreuzenden kulturellen Einflïsse zeigen sich auch auf diesem engen Gebiete und lassen diese paränetisch-panegyrische Literatur als ein buntes Gewebe verschiedenartiger Fäden erscheinen, die zu sondern und auf ihren Ursprung zurückzuverfolgen eine lockende Aufgabe literarhistorischer Forschung ist. Das uralte, nie vergessene Gedankengut des antiken Topos $\pi \varepsilon \rho i ~ \beta a \sigma \iota \lambda \varepsilon i \alpha s$, die Vorschriften der griechischen Rhetorik für die Lob- und Grabrede im allgemeinen und sonstige Reminiszenzen aus altgriechischer Lektüre, Erörterungen im AnschluB an Sätze der Bibel und christlicher Theologen und neue Erwägungen, wie sie die besonderen Verhältnisse von Byzanz oder die individuelle Natur des jeweils vorliegenden Falles eingeben, vereinigen sich zu einem vielfarbigen Bilde, in welches im elften

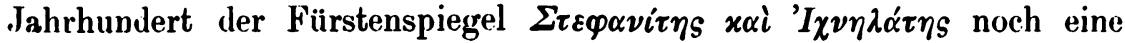
neue, orientalische Nuance hineinträgt. Es würle sich wohl verlohnen, einmal diese ganze Literatur zum Gegenstande einer besonderen Studie zu machen, bei der sich zeigen würde, daB, was bei diesen Produkten der „Klassizismus" entbehrt, der „Historismus“ gewinnt, und daB in denselben die verschiedene Eigenart der einzelnen Autoren, Kreise und Perioden in interessanter Weise sich widerspiegelt, besonders in Hinsicht auf das wechselnde Verhältnis zur Antike. Auch würde eine solche Untersuchung in wünschenswerter Weise zur Aufklärung darüber beitragen, welche antiken Schriftsteller sich in Byzanz der besonderen Beriicksichtigung der verschiedenen Verfasser und Zeiten erfreuten. ${ }^{1}$ )

1) Bei antikem Material ist selbstverständlich immer nach Möglichkeit festzustellen, ob es direkt aus antiker Quelle geschöpft oder durch das Medinm eines 
Die Vorarbeiten für eine solche zusammenhängende Darstellung dieses Literaturzweiges verlangen die Beteiligung vieler. Zufällige Beobachtungen bei der Lektüre antiker und byzantinischer Autoren, Sammlungen für einzelne Gedanken und Zitate u. dgl. lassen manchen Zusammenhang erkennen, der sich auch der eifrigsteu ad hoc unternommenen Nachforschung vielleicht lange entzogen hätte. Darin liegt die Berechtigung begrïndet, hier mit kleinen Einzelbeitrügen hervorzutreten, wie ein solcher im Folgenden gegeben werden soll.

Unter den von A. Heisenberg vor kurzem herausgegebenen kleinen Schriften des Georgios Akropolites ${ }^{1}$ ) findet sich auch eine Grabrede auf Kaiser Johannes Dukas. Es macht dem Geschmack des Verfassers Ehre, daB er eine frostige Anhäufung der stereotypen Sätze des antiken Topos, wie sie uns bei anderen begegnet, vermieden hat, eine Enthaltung, die man dem gelehrten, in der alten Literatur wohlbelesenen Mann doppelt hoch anrechnen muB. An Berührungen mit jenem Topos fehlt es freilich nicht. Aber sie betreffen zum gröBten Teile Gedanken, die infolge ihrer weiten Verbreitung kaum als Eigentum des Königstopos und der antiken Literatur überhaupt empfunden werden, oder die so allgemeinen und naheliegenden Inhaltes sind, daB sich schwer abschätzen läBt, wieweit unmittelbar oder mittelbar, bewuBt oder unbewuBt antike Vorbilder eingewirkt haben und wieweit der Verfasser den betreffenden Gedanken selbständig aus sich erzeugt hat. So begegnet uns S. 14, 24 ff.; 26,14f. die seit Alkaios an zahllosen Stellen durchgeführte Verbildlichung des Staates durch das Schiff und seines Regenten durch den Steuermann, S. 29, 6 ff. die Vergleichung des Herrschers mit der Sonne (s. auch S. 12, $13 \mathrm{f}$, , wo aber der Gedanke in die

andern Byzantiners hindurchgegangen ist. So ist es z. B. charakteristisch, daß in dem ron B. Wassiliewsky und V. Jernstedt mit dem Strategikon des Kekaumenos (Petersburg 1896) herausgegebnen anonymen Königsspiegel von den beiden in Altertum spielenden Anekdoten S. 100, $26 \mathrm{ff}$. die erste, von Augustus und Athenodoros handelnde sicher zunächst einer byzantinischen Quelle entnommen ist (vgl. die von mir B. Z. V [1896] S. 497. 499 besprochenen Texte), die zweite, die das Freundschaftsverhältnis zwischen Algaros und Augustus betrifft, wohl ron Haus aus byzantinischer Quelle (Prokop Pers. 2 S. 117 d. Paris. Ausg.) entstammt, da erst durch die christliche Legende die Aufmerksamkeit anf Abgar gelenkt wurde. Das stimmt sehr gut zı der ganzen Art dieses Königsspiegels, der trotz mancher Berührungen mit dem Stoffe der antiken Traktate ïber das Königtum (z. B. S. 93, 1f.; 93, 9f. [dies wohl aus byzantinischer Mittelquelle, vgl. Agapet 1]; 98, $15 \mathrm{ff}$; 98, $21 \mathrm{ff}$; 99, $29 \mathrm{ff}$; 100, $24 \mathrm{f}$.) doch einen Verfasser verrät, der in seiner Zeit wurzelt.

1) Georgii Acropolitae opera rec. Aug. Heisenberg. Vol. alterum. Lipsiae 1: 03. Die Grabrede S. 12-29. 
hiblische Sphäre übergeleitet ist) $)^{1}$ ), S. 21, 15 die Bezeichnung des Fürsten als Vaters seiner Untertanen. ${ }^{2}$ ) $\mathrm{DaB}$ der wahre König nicht im Genub, sondern in Mühe und Arbeit seine Aufgabe erblickt (S. 15, $23 \mathrm{ff}$.) $\left.{ }^{3}\right)$, daB er Verbrechern, insbesondere solchen, die sich gegen ihn vergangen haben, Milde angedeihen läßt (S. 21, $26 \mathrm{ff}$; $\left.22,28 \mathrm{ffi})^{4}\right)$, daB er menschenfreundliche, nicht das Volk bedrückende Beamte einsetzt (S. 27, $14 \mathrm{ff}$. $)^{5}$ ), sind nie fehlende, vielfach in Form stehender Kapitel ausgeführte Erwägungen der Fürstenspiegel und verwandter Literatur. Aber was Akropolites hierüber vorbringt, trägt nirgends den sichern Stempel der Entlehnung.

Eine greifbarere Beziehung zu dem traditionellen Gedankengut jener Traktate liegt S. 27, 32 ff. zutage. Nachdem im Vorhergehenden

1) Aus der antiken Literatur vgl. etw Dion Rede 3, $73 \mathrm{ff}$; 1, 24; Plut. $\pi$ o. $\dot{\eta} \gamma \varepsilon \mu$. $\alpha \pi \alpha i \delta .5,2$ S. 955, $18 \mathrm{ff}$; Themist. Rede 11 S. 150 b Pet., 13 S. 11 li7 a, aus

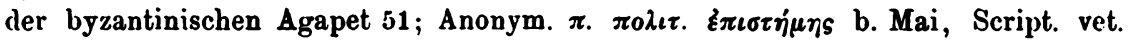
nov. coll. II (Rom 1827) S. 602 (fol. 299 a 24 ff.). S. auch Seren b. Stob. Floril. 47, 20. Näher geht auf den Gedanken ein Fischer, De Dion. Chr. or. III. comp. et font. (Bonn 1901) S. $38 \mathrm{f}$.

2) Vgl. z. B. Xenoph. Kyrup. 8, 1, 1; (Xen. Ages. 7, 3 ;) Cic. de rep. 1, 35, 54;

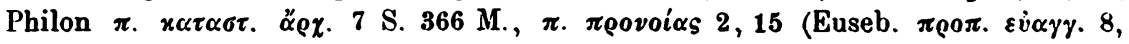
14, 3); Sen. de clem. 1, 14; Dion Chrys. Rede 1, 22; 3, 5; Themist. Rede 11

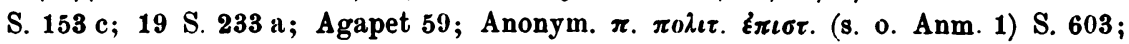
Basil. $x \neq \varphi$. $\pi \alpha \rho \alpha \iota v \varepsilon \tau$. 28; Konst. Man. Chron. 6187. Von den Beamten Theophyl. Rede an Alex. Komn. 8 S. 556 d. Dahin gehört auch die beliebte Anführung von Hom. Od. 2, 47. 234 mit Beziehung auf den Herrscher, wie Themist. Rede 1 S. 17 a ; Sopat. b. Stob. Flor. 46, 55 S. 225, 7 ff. Mein.; Thom. Mag. b. Mai, Script. vet. nov. coll. III (Rom 1828) S. 154, 20; Georg. Kypr. Lobr. auf Mich. Palaiol., Mign. Patr. Gr. 142 Sp. 385 b. Die Homerstelle ist wohl auch berïcksichtigt bei Herod. 3, 89 (ïjiıs).

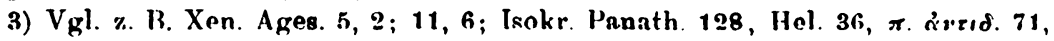
Brief 7, 4; Cass. Dion 36, 9, 2; Themist. Kede 6 S. 75 a.

4) Finige Beispiele aus der unübersehbaren Zahl von Stellen habe ich B. Z.

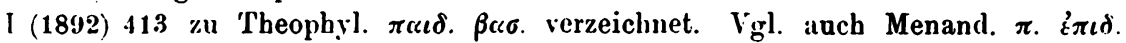
S. 37f, $29 \mathrm{f}$. Christlicher Hinweis auf die als Entgelt zu erwartende Milde Gottes (Akr. 22, $32 \mathrm{ff}$ ) Agapet 8. 23. (44.) 64 mit den Parallelen im Roman Barlaam und Joasaph (B. Z. II [1893] 446. 448f.); Nikeph. Blemm. Königsspiegel 3 S. 620 b Migne.

5) Isokr. $\pi$ òs Nı\%oxh. 16, 20; Xenoph. Kyrup. 8, 1, 9 ff.; Jul. Rede 2 S. 117, 6 ff. Hertl.; Thenist. Kede 5 S. 67 a f.; 8 S. 117 ad; 118 c f.; Synes. Rede 1. 30; Prokop Rede a. Anast. S. 37 Vill.: Chorik. Rede a. Ar. u. Steph. 1, ; (Revue de philol. 1877 S. 65); 'Theophyl. $\pi \alpha \iota \delta$. Buб. 20; Nikeph. Blemm. Königsspiegel 11; Georg. Kypr. Iobr. auf Andron. Palaiol. S. 412 b Migne (vielleicht durch Akr. beeinfluBt; $i v \mu \varepsilon \dot{\omega} v \ddot{\varkappa} \nu \vartheta \rho \omega \pi 0 s$ hat auch Akr. 27, $17 \mathrm{f}$.). Vgl. auch Men. $\pi$. $\{\pi \imath \delta \varepsilon / x \tau$. S. $\mathbf{3 7 5}, 18 \mathrm{ff}$. (s. im Texte weiter unten). Verant,rortlichkeit des Herrschers für die Taten dor Beamten: [Isokr.] $\pi \rho . \Delta \eta \mu \dot{\nu} .37$; Isokr. $\pi \rho$.

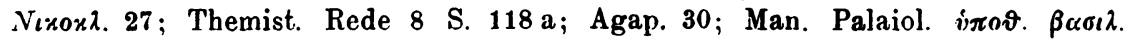
$\dot{c} \gamma \omega \gamma \cdot 76$ 
die Beschäftigung des Kaisers mit Philosophie und Wissenschaften

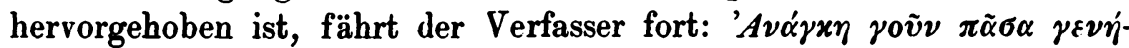

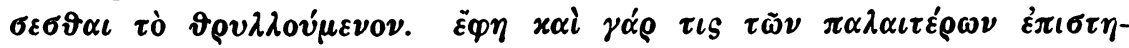

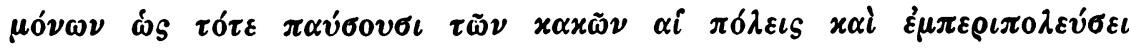

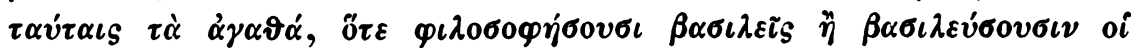

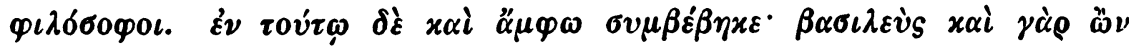

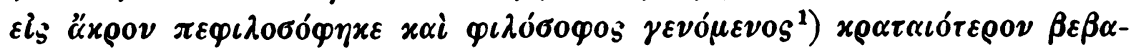

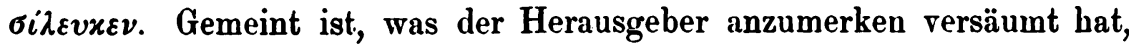
Platon $\pi 0 \lambda \iota \tau \varepsilon i \alpha$ S. $473 \mathrm{~d}^{2}$ ) Es wird nicht ohne Nutzen sein, dieses Zitat im Zusammenhang mit anderen Anführungen der Platonstelle etwas näher ins Auge zu fassen. Unter den ungemein häufigen Erwähnungen der platonischen Worte und Anspielungen auf dieselben ${ }^{3}$ ) sondern wir zunächst diejenigen aus, die ein einfaches, von keiner $\mathrm{Zu}$ stimmung oder Ablehnung begleitetes Referat über die platonische These enthalten. Hierher gehören Apul. de Plat. et eius dogm. 2, 24 S. 100, 25 Goldb.; Albinos (nach d. Überl. Alkinoos) $\varepsilon i \sigma \alpha \gamma .34$; Lactant. div. inst. 3, 21. Über zustimmende ${ }^{4}$ ) Verwendung der Stelle durch einen Späteren referiert Iul. Capitol. M. Ant. phil. 27, 7. Der gleiche Zusammenhang mag obwalten an der verstüınmelten Stelle Rutil. Lup. de fig. sent. 1, 6. Eine Polemik gegen den platonischen Satz führt Themist. Rede 8 S. 107 c Pet., als Anknüpfungspunkt für die Forderung einer Vereinigung praktischer Fähigkeit mit historiographischer Betätigung benutzt ihn Polyb. 12, 28, 2 ff. Die übrigen Stellen, soweit sie mir bekannt sind, lassen sich in drei nach den Stichworten Zukunft, Vergangenheit, Gegenwart trennbare Gruppen zerlegen.

I. Der von Platon geforderte Zustand erscheint als ein zu erstrebendes $\mathrm{Ziel}$, indem die Worte des Philosophen mit ausdrücklicher oder aus dem Zusammenhang sich ergehender Zustimmung angefïhrt oder angedeutet werden: Valer. Max. 7, 2 ext. 4; Justin Mart. Apol. 3; Aristid. Rede 46 S. 206, 1 ff. Dind. (zustimmend S. 207, $1 \mathrm{f}$ ); Greg. Naz. Brief 24 (sonst 140) S. 22 d. Maur. Ausg.; Synes. Rede 1, 32

1) Heisenberg behält das überlieferte $\varepsilon v \eta \sigma o ́ \mu \varepsilon v o s$ bei; dasselbe scheint mir aber mit dem Zusammenhang unvereinbar.

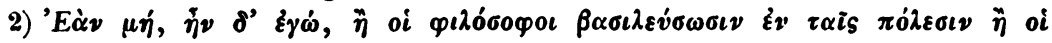

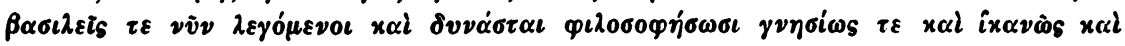

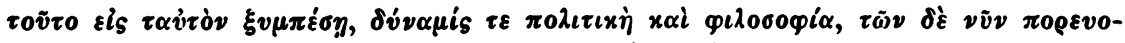

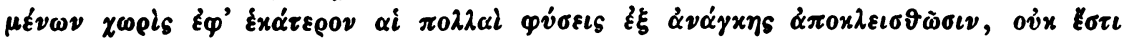

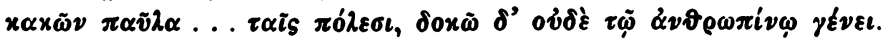

3) Bei welchen auch verwandte Stellen wie Plat. Brief 7 S. 326 a f., 328 a, 335 d.; 2 S. 310 e; Republ. 6 S. 487 e; Ges. 4 S. 711 ef. gelegentlich mit hereinspielen.

4) $\mathrm{DaB}$ es sich um eine solche bandelt, zeigt der Zusammenhang. 
S. $32 a^{1}$ ); Syrian zu Hernog. II S. 17, 6 ; Boëth. de cons. philos. 1, $4^{2}$;

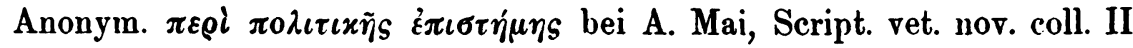
(Rom 1827) S. 608 (vgl. auch S. 602); Thomas Mag. ebenda III (Rom 1828) S. 172 a. E.

II. Platons Forderung soll zu einer gewissen Zeit der Vergangenheit ihre Verwirklichung gefunden haben: Aristot. Rhetor. 2, 23

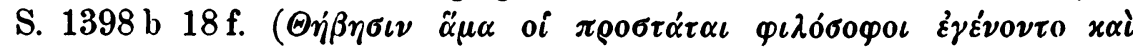

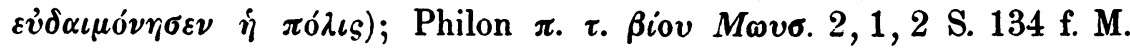
200, 9 ff. C. von Moses; Plut. Numa 20 von Numa (s. auch Plut. Dion 1, Vergl. von Demosth. u. Cic. 3); Aurel. Vict. Caes. 15 (von Antoninus

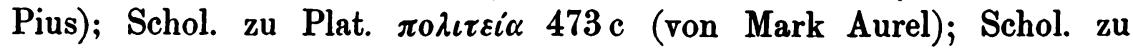
Aristeides S. 266 Fromm. (von den durch Aristeides verteidigten vier athenischen Staatsmännern); Leben d. Joh. Bat., B. Z. XIV (1905) S. $230,15 \mathrm{ff}^{3}$ )

III. Die platonische Forderung wird als in der Gegenwart des zitierenden Verfassers verwirklicht bezeichnet: Cicero ad Quint. fratr. 1, 1, 10, 29; Themist. Rede 2 S. 40 a Pet.; 17 S. 214 a; derselbe in einem an Kaiser Julian gerichteten Schreiben nach Julians Antwort S. 329, 2 Hertl.; Prudent. in Symm. 1, 30 ff.; Agapet Fürstensp. 17; Georg. Kypr. Enk. auf Andr. Palaiol. Sp. 413 a Migne. ${ }^{4}$ ) In diese Kategorie gehört auch unsere Akropolitesstelle.

Aus dieser Zusammenstellung ergibt sich, wenn nicht mit Sicherheit, so doch mit der hier allein erreichbaren hohen Wahrscheinlichkeit folgendes: Die Verwendung der Platonstelle bei Akr. beruht nicht auf einer Reminiszenz aus eigener Platonlektüre; die Analyse hat vielmehr diese Stelle von denjenigen zu scheiden, die dem Verfasser sein Studium antiker Philosophen an die Hand gab. Die Platonstelle war schon

1) Zum Gedanken vgl. auch 1, 6 S. 6 c; 7 S. 7 b (= $=\pi$.

2) Darnach Ioann. Sarisber. Pol. 4, 6 g. E.

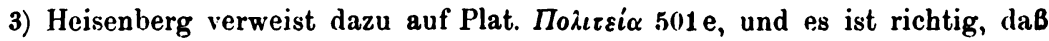
die Vita speziell mit dieser Stelle insofern stimmt, als sie nur von der Regierung des Staates durch Philosophen, nicht davon spricht, daB die Philosophen Könige, die Könige Philosophen werden sollen. Allein es ist kaum anzunehmen, daß der Verfasser eine andere als die berübmte oft zitierte Stelle im Auge hatte, deren Nachwirkung vielleicht in den allerdings vom Verfasser als eigenem Zusat\%

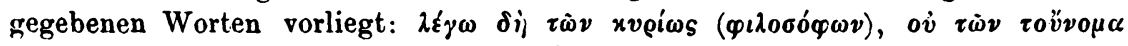
$\psi \varepsilon v \delta \circ \mu \varepsilon \dot{v} \omega \nu$ (Platon:

4) Die Stelle steht hier zwischen anderem Platonischen; das Vorausgehende nach Ges. 4 S. 709e (vgl. 710 c), im Nachfolgenden (413 h) ist. das von Platon Theait. 144a, Republ. $503 \mathrm{c}$ Ausgeführte stillschweigend berïcksichtigt. Die ťbereinstimmung in der Wiedergabe der Stelle ron den philosophischen Königen mit $A \mathrm{kr}$. ist nicht derart, daB sie nicht auf Zufall beruhen könnte. 
längst mit dem Königstopos verbunden und wurde in diesem Zusammenhange von Akr. übernommen. Suchen wir den Weg, auf welchem das Zitat $z u$ ihm gelangte, genauer zu bestimmen, so läßt sich ein uns bekannter Schriftsteller, soweit ich sehe, als Quelle nicht namhaft machen ${ }^{1}$, wohl aber im allgemeinen die Vermutung aufstellen, daB nicht eine philosophische Ausführung über das Königsideal, sondern ein Stück der rhetorischen Literatur, nicht ein Königsspiegel im eigentlichen Sinne des Wortes, sondern eine Lobrede auf einen Fürsten die Vorlage gewesen ist. ${ }^{2}$ ) Das ergibt sich schon aus der panegyrischen Verwendung der platonischen Worte, die nur in einem Enkomion oder dem enkomiastischen Teile eines Epitaphios am Platze ist. Es spricht dafür ferner, daß diese Art der Verwendung durch die unter III genannten rhetorisch gebildeten Schriftsteller, zu denen jedenfalls eine Anzahl anderer verlorener oder mir unbekannter Benutzer der Platonstelle hinzuzufügen wäre, bereits eingeführt war. Eine Bestätigung liegt endlich in den engen Beziehungen, die auch sonst zwischen der vorliegenden Rede des Akr. und der antiken Rhetorik vorhanden sind. Heisenbergs Ausgabe wird hoffentlich zu einer Arbeit über Sprache und Stil des Akropolites den AnstoB geben, in welcher auch dieser Punkt seine erschöpfende Behandlung finden wird. Für den Augenblick genügt es, meine Behauptung durch eine keinen Anspruch auf Vollständigkeit erhebende Sammlung solcher Stücke aus dem Apparat der antiken Rhetorik zu begründen, welche in unserer Grabrede Verwendung gefunden haben.

I. Disposition. Für diesen Punkt hat sich zwar Akr. in der Hauptsache nicht an die rhetorische Schablone gehalten. ${ }^{8}$ ) Wenn

1) Was Akr. mit don moiaten nicht nur der unter III, kondern uuch dor unter I und II genannten Autoren in der Wiedergabe der Platonstelle gemein bat, liefert für die Quellenkritik keinen Anhaltspunkt: alle bieten von den platonischen Worten gerade das, was sich jedem Leser жunïchst einpriigt. DaB 'Themistios Akr. bekannt war, lïBt sich aus der Byz. \%. XIV (1905) 258 besprochenen Tatsache wahrscheinlich machen; für die Platonstelle ist er aber nicht die Quelle gewesen, es sei denn, daB Akr. durch ihn die Anregung $\approx n$ dem Zitate empfing und das von Themistios Angeführte aus Platon ergänzte.

2) Natürlich bleibt auch die Möglichkeit, daB das Zitat in eine rhetorische Anweisung für Lobreden Eingang gefunden batte und daraus von $\mathrm{Akr}$. übernommen wurde.

3) Wie dies z. B. von Julian in dem Enkomion auf Konstantius, z. T. auch in dem auf Eusebia, und von Prokop in der Lobrede auf Anastasios (über diese K. Seitz, Die Schule v. Gaza [Heidelberg 1892 Dissert.] S. 48) geschehen ist. Auch Georgios v. Kypros lehnt sich in seinen Lobreden auf Michael und Andronikos Palaiologos wenigstens teilweise an die traditionelle Theorie, deren Berücksichtigung er Sp. 389 b Migne ausdrïcklich bekennt mit den Worten: $\Pi \alpha \tau \rho i \delta \alpha$ 
S. 25, $3 \mathrm{ff}$. vom Enkomion zum Threnos übergegangen wird und von Kap. 18 an auf die Klage der Trost folgt, so entspricht allerdings beides den bei Menand. $\pi$. $\dot{\varepsilon} \boldsymbol{\pi} \iota \varepsilon \iota x \tau$. S. 421, 10.15 Sp. gegebenen Vorschriften; aber der Threnos ist nur angedeutet, nicht, wie es die rhetorische Theorie verlangt, ausgeführt, und die Beendigung der ganzen Rede durch Trosteszuspruch liegt so sehr in der Natur der Sache, daB sich darauf noch keine Schlüsse auf Abhängigkeit von der rhetorischen Theorie bauen lassen. Schon näher kommen wir der letzteren, wenn wir den im enkomiastischen Teil enthaltenen Abschnitt über die Tugenden des Kaisers S. 21, $13 \mathrm{ff}$. prüfen. Zwei Tugenden treten hier in den

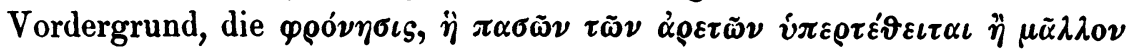

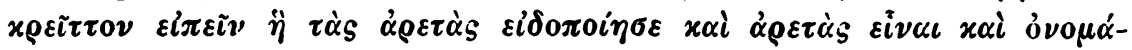
$\left.\zeta \varepsilon \sigma \vartheta \alpha \iota \pi \varepsilon^{\prime} \pi \varepsilon \iota \varepsilon^{1}\right)$ (S. 21, 20 ff.), und eine zweite, die S. 21, 14.15. 24. 25

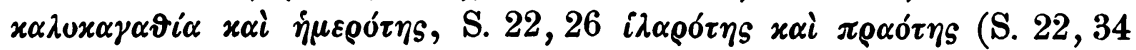

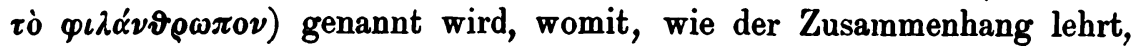
die Milde gegen Schuldige gemeint ist. Beide Tugenden sind auch bei Menander S. 373, $11 \mathrm{ff} ; 374,27 \mathrm{ff}$. eingehend berücksichtigt, die

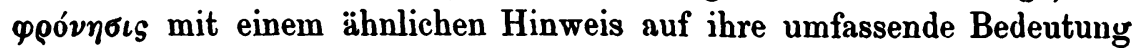
$(373,9 \mathrm{ff}$; 376, $18 \mathrm{ff}$.). Erwähnung verdient auch, daB S. $27,14 \mathrm{ff}$. wie bei Menander S. 375, $18 \mathrm{ff}$. die Entsendung guter, nicht räuberischer Beamten unter den Gesichtspunkt der Gerechtigkeit des Fürsten gerückt wird. Wichtiger ist, daB sich Akropolites in der den SchluB des Ganzen bildenden Lobrede auf Theodoros II Laskaris wenigstens andeutungsweise mit der von der Theorie vorgeschriebenen Einteilung des Enkomions bekannt zeigt, zu deren eingehender Verwendung ihm allerdings dieser nur akzessorische kurze Teil seiner Grabrede keinen Raum bot. S. $28,12 \mathrm{ff}$. wird der neue Kaiser mit den Worten charak-

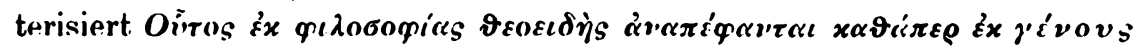

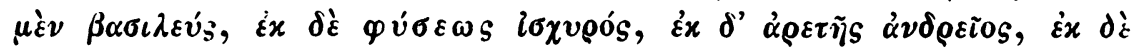
$\tau \tilde{\omega} \nu \pi \varrho \alpha \gamma \mu \alpha^{\alpha} \tau \omega \nu \pi \alpha \nu \dot{c}^{\prime} \varrho \iota \tau \tau$ s. Die stehenden Gesichtspunkte der Lobrede und rerwandter Reden und Redeabschnitte sind bei Menander S. 370, 11; 371, 15. 18; 41:3, 10 f.; 419, 17; 420, 11 ff. 25; Dionysios

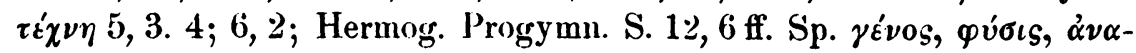
$\tau \varrho \circ \varphi \eta^{\prime}(\dot{\alpha} \gamma \omega \gamma \eta \dot{)}), \pi \varrho \alpha \dot{\xi} \xi \iota \varsigma$, wozu sich freilich je nach den Umständen noch weitere l.unkte gesellen. Akropolites hat also dieses Schema bis

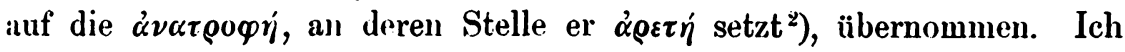

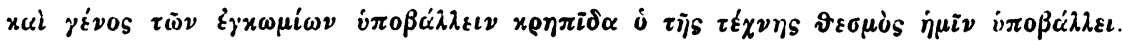

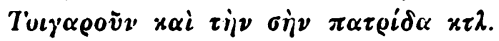

1) $\pi \dot{\varepsilon} \pi$ o $x \varepsilon$ ? Zur Form vgl. J. Psichari, B. Z. IX (1900) 65y. Über die Formulierung des Gedankens s. unten S. $489 \mathrm{f}$.

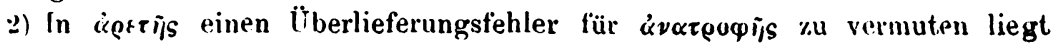


füge noch bei, daB, was die einleitenden Wendungen für die einzelnen Teile der Rede betrifft, Akr. S. 13, $16 \tau i_{S}^{\prime} \mu o \iota \delta \alpha \nu \varepsilon i \sigma o \iota ~ \gamma \lambda \tilde{\omega} \tau \tau \alpha \nu$ 'O $\mu \eta^{\prime}-$

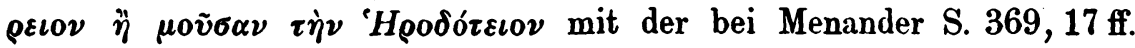

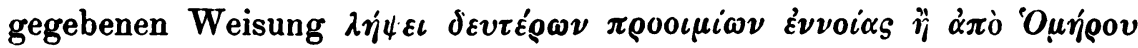

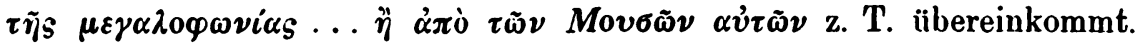

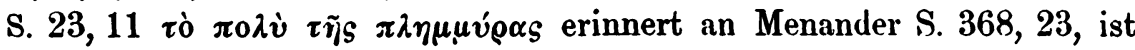
aber anders zugespitzt und steht an anderer Stelle. ${ }^{1}$ )

II. Kunstmittel rhetorischer Darstellung.

a. Synkrisis (bezw. $\left.\alpha \nu \tau \iota \pi \alpha \rho \alpha \beta o \lambda \eta^{\prime}\right)$. Dieselbe hat nach Menander S. 376, $31 \mathrm{ff}$; 417, $5 \mathrm{ff}$; 421, $4 \mathrm{ff}$. (vgl. auch Theon Progymn. S. 112, $20 \mathrm{ff}$.) in der Weise zu geschehen, daB der Gerühmte in bezug auf seinen gesamten Charakter oder auf einzelne Vorzüge mit anderen Trefflichen verglichen und dabei gezeigt wird, daB er dieselben ïberragt. ${ }^{2}$ ) Solche Synkriseis hat Akr. S. 22, 27 f.; 23, $16 \mathrm{ff}$. $21 \mathrm{ff}$. (Johannes Dukas hat länger regiert als Titus, vgl. Theon Progymn. S. 113, $9 \mathrm{f}$.); 24, $6 \mathrm{ff}$. (ausgebreiteterer Nutzen, vgl. Theon Progymn. S. 113, $8 \mathrm{f}$.); 28, $15 \mathrm{ff}$.

b. Zitate aus der klassischen Literatur: S. 24, $33 \mathrm{ff}$. (Hom. Il. 2, 489; vgl. auch Macrob. Sat. 6, 3,6); 25, 10 f. (Hom. Il. 13, 636); 25,

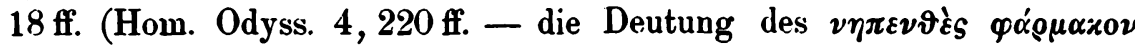

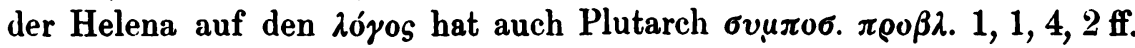
S. 743, $37 \mathrm{ff}$; daB sie geeignet war, auch in die Rhetorschule Eingang zu finden, liegt auf der Hand); 27, 22 (der Thesaurus unter "̈ $\pi \iota$ s notiert Tryphiod. 5!8, die Wendung wird aber wohl auch bei anderen Dichtern vorkommen); 28, $6 \mathrm{ff}$. (Hom. Il. 2, $478 \mathrm{f}$; die Stelle wird, allerdings in anderem Zusammenhang, in der rhetorischen Literatur häufig verwertet; s. den Index auctorum b. Spengel. Vgl. auch Plut. $\pi$. $\tau$. 'A $A \varepsilon_{\xi}^{\xi}$.

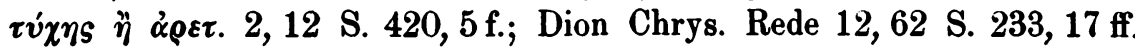

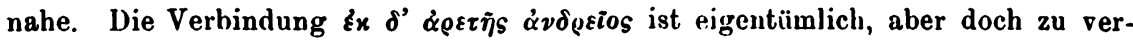

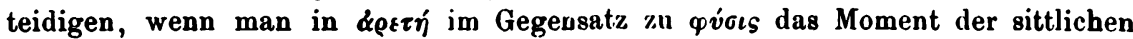
Arbeit des Menschen an sich selbst betont.

1) Das $\pi \dot{\varepsilon} \lambda \alpha \gamma o s$ verwendet im Sinne der Stelle bei Menander Prokop Lobr. a. Anast. S. 33 Vill. Auch Georg. Kypr. hat das Bild, «. B. Lobr. auf Georg. Prol. 1 Sp. 301 a Migne, auf Mich. Pal. Sp. 373a, auf Andr. Pal. Sp. 389a. Zu Menand. S. 369, 8 ff. stimmt Prokop S. 42.

2) Die Regel der Schule ist in der rhetorischen Praxis sehr eifrig befolgt worden und gehört zu den Stürken des Schulapparates, die am frühesten nachweisbar sind. Vgl. z. B. Isokr. Euag. 35 ff. 65 (s. dazu Chorik. Red. a. Ar. und Steph. 5, 6 [Revue de philol. 1877 S. 72]); Sen. de clem. 1, 11; Themist. Rede 4 S. 57 c; 58 a f.; 5 S. 65 bf.; 66 a f.; 67 a; 6 S. 74 cf.; 7 S. 94 b; 97 b f.; 8 S. $110 a$ f.; 10 S. 130 a f.; 13 b b f; 140 a f.; 11 S. 145 b; 15 S. 193 c f.; Prokop, Rede a. Anast. S. 36 f. Vill.; 39 f.; 44; Chorik. Rede a. Ar. n. Steph. (Rev. de philol. 1877 S. 84) Vgl. auch P. Wendland, Hermes 39 (1904) S. $536 \mathrm{f}$. 
Dind.); 28, 10 (Herod. 1, 65; vgl. Galen Protr. 9 S. 13, 1 ff. Kaib.; Themist. Rede 7 S. $97 \mathrm{bcd} ; 15$ S. $193 \mathrm{c} ; 19$ S. 225 d). ${ }^{1}$ ) Eine ho-

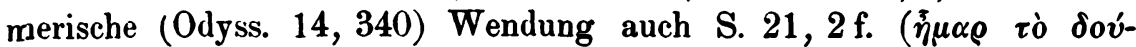
$\lambda_{\imath}(o v)$. Wie Akr. zu der eigentümlichen Verwendung des Niobemythus S. 13, 4 f. gekommen ist, habe ich bis jetzt nicht feststellen können; droch darf immerhin die Möglichkeit angedeutet werden, daB die $\mathrm{Be}$ hrandlung des Mythus in einem rhetorischen Lehrbuche, wie sie z. B. beei Nikol. Progymn. S. 304 ff. (Walz, Rhet. gr. I) vorliegt, Akr. oder einem Vorgänger den Gedanken eingegeben hat. In der Kataskeue mochte etwa die von Akr. S. 13, 5 ff. erwähnte Wirkung des Schmerzes auf den Menschen zugunsten des Mythus ins Feld geführt sein. Weitere Stücke antiken Apparates, die nicht auf eine bestimmte

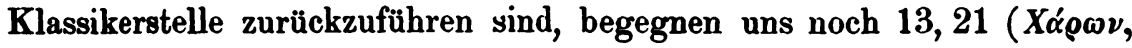
doch waltet schon die im Neugriechischen herrschende ${ }^{2}$ ) Vorstellung von Charon nicht als Totenfährmann, sondern als Vertreter des Todes und

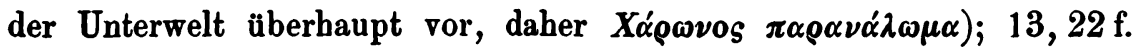

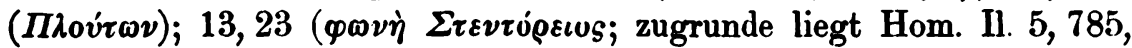
es handelt sich aber um einen sprichwörtlichen Ausdruck).

c. Bilder und Vergleiche: S. $12,4=28,20 \mathrm{f}$; 12,5 (vgl. Menand. S. $377,16 \mathrm{f}$.) $; 12,7=28,17 ; 12,24 \mathrm{ff}$. $=28,30 \mathrm{ff}$. Über den Schiffsund Sonnenvergleich sowie die Stellen 12, $9 \mathrm{ff}$; $29,3 \mathrm{ff}$. ist bereits oben gesprochen.

d. Ekphraseis: die Schilderungen des Winters und Sommers S. 17, $10 \mathrm{ff}$., insofern besonders gute Beispiele dieses rhetorischen Kunstmittels ${ }^{3}$ ), als die Schilderungen durch den Zusammenhang in gar keiner Weise gefordert waren, also nur um des rhetorischen Effektes willen eingefügt wurden.

e. Wörter poetischer oder rhetorischer Färbung: 12,4 ( $\pi \dot{v} \varrho \gamma \omega \mu \alpha)$;

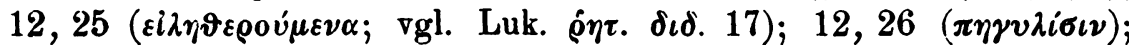

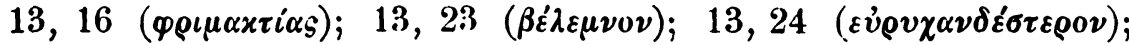

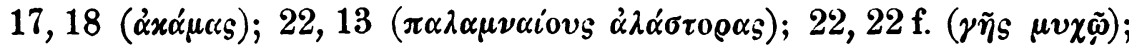

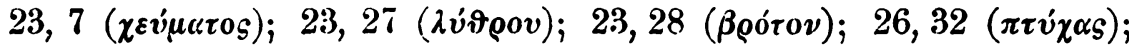

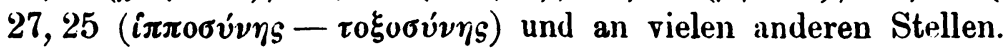

f. Häufungen. ${ }^{4}$ ) Das Streben, der Rede durch Koordinierung von

1) Ich komme auf diese Stelle unten zurück.

2) Vgl. O. Waser, Charon, Charun, Charos S. $87 \mathrm{ff}$.

3) Näheres über dasselbe und die darüber handelnde Literatur bei W. Schmid, Der Attizismus in seinen Hauptvertretern II (Stuttgart 1889) S. 268; E. Norden, I. antike Kunstprosa (Leipzig 1898) S. $285 \mathrm{f}$.

4) Vgl. darüber W. Schmid, Der Attizismus, an den im Register unter „Ḧ̈ufung der Begriffe" verzeichneten Stellen; H. v. Arnim, Leben und Werke d. 
2,wei oder mehr Ausdrücken die Eigenschaft des $i \psi \cdot \gamma \lambda \dot{v} \nu$ und $\mu \varepsilon \gamma \omega \lambda$ $\left.\pi \varrho \varepsilon \pi \varepsilon^{\prime}{ }^{1}\right)$ zu geben, zieht sich durch den ganzen Epitaphios hindurch und bestimmt wesentlich seinen stilistischen Grundcharakter. Beispiele finden sich fast in jeder Zeile. Ich analysiere nur den Anfang der

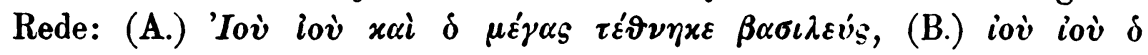

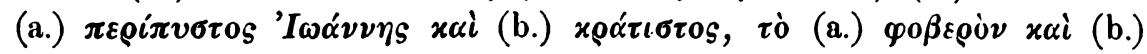

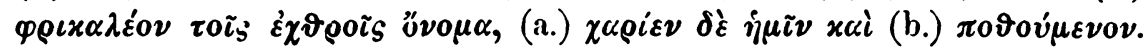

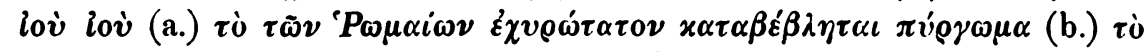

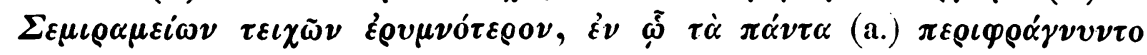

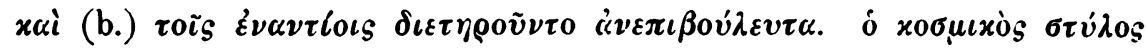

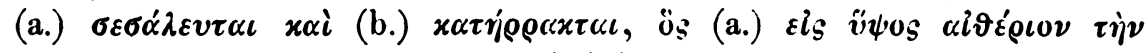

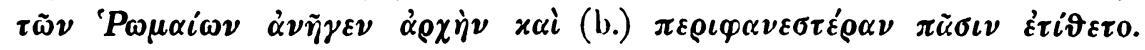
Man vergleiche etwa noch 16, $23 \mathrm{ff}$; $17,6 \mathrm{f}$; 17 , $18 \mathrm{f}$; 1 i, $25 ; 17,27 \mathrm{f}$; 17,$30 ; 17,33 \mathrm{f}$; 18,$2 ; 19,1 \mathrm{f}$; 19, $5 ; 19,9 \mathrm{f}$; 19, 25; 24, 7; 24, 8 f.; 24, $9 \mathrm{f}$; 24, $23 \mathrm{f}$. Gleicher Tendenz verdankt wohl auch die bei Akr.

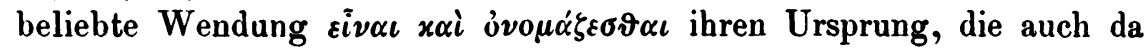
angewandt wird, wo es auf eine Scheidung der beiden Begriffe sachlich nicht ankommt. Zu den B. Z. XIII (1904) 527 Anm. 1 gesammelten

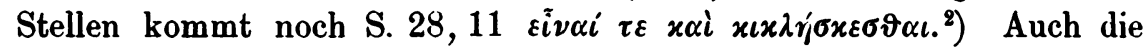
Koordinierung von drei und mehr Gliedern ist häufig; drei finden sich z. B. $14,24 \mathrm{ff}$; $15,29 \mathrm{f}$; $15,31 \mathrm{ff}$; 15, 35ff.; $16,18 \mathrm{ff}$; $16,27 \mathrm{ff}$; $18,16 \mathrm{f}$; $18,24 \mathrm{f}$; $18,26 \mathrm{ff}$; $21,4 \mathrm{ff}$; 21, $17 \mathrm{f}$; $23,30 \mathrm{ff}$; $27,19 \mathrm{ff}$; 27,$30 ; 28$, $33 \mathrm{f}$; ;ier $(2+2) 17,2 \mathrm{ff}$; $18,1 \mathrm{ff}$; fünf bezw. sechs und mehr 14, $3 \mathrm{ff}$.

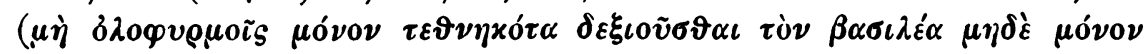

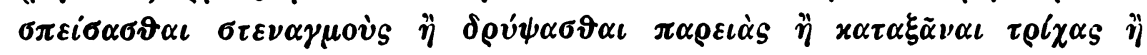

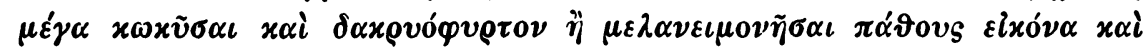

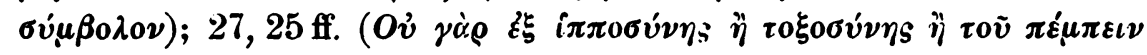

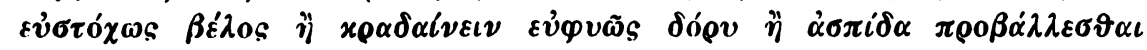

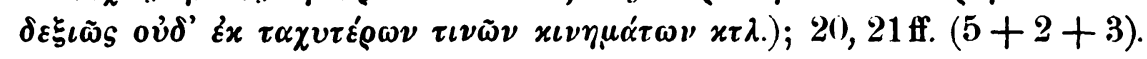
Mit solchen Häufungen stehen im Znsammenhang die zahlreichen

g. Polysyndeta, wofür die unter $f$ gesammelten Stellen genügend Beispiele enthalten. Als Gegenstück ist das

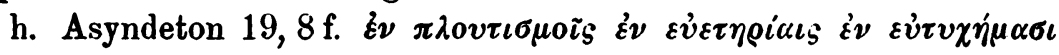
anzuführen. Die hier stattfindende Wiederholung der Präposition leitet uns zu den ungemein häufigen Beispielen der

i. Anaphora: $12,1.3$ (22); 12,14 f.; 13,12 f.; 13, 23. 26; 16,14 f.;

Dio v. Prusa (Berlin 1898) S. 459; K. Praechter, Hierokles der Stoiker (Leipzig 1901) S. $95 \mathrm{ff}$.

1) Vgl. v. Arnim a. a. 0.

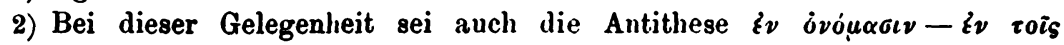
$\pi \rho \dot{\alpha} \gamma \mu \alpha \sigma \iota$ S. 28, 23 f. nachgetragen. 
18,3 f.; 18, 5 (vgl. 19, 16; 20, 3); 18, 34; 19, 1 (vgl. 22, 34 f.); 20, 12 f.; 23,$1 ; 24,22$ f.; 25,12 f.; 25, 15; $25,30=27,30$ f.; 26, 9 ff.; 26, 31;

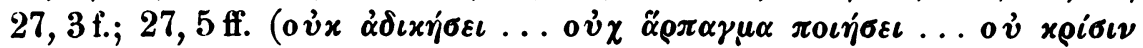

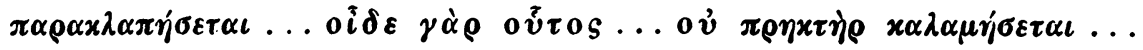

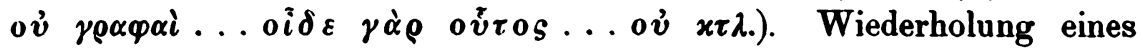

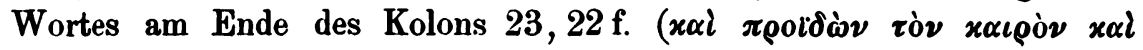

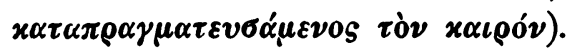

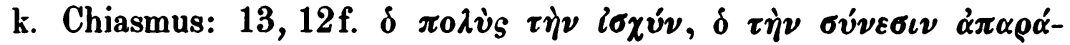

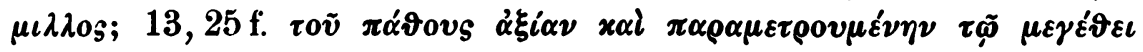

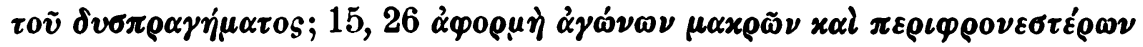
E̊

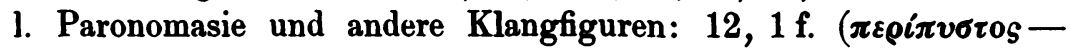

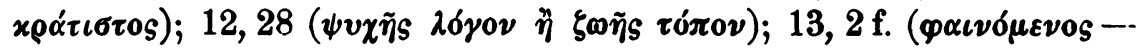

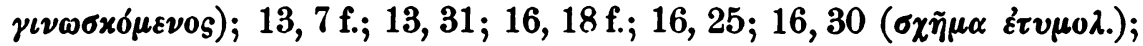
17,23 f.; 17, 33 f.; 18, 2; 18, 5 f.; 18, 18 f.; 18, 34 f.; 19, 23; 19, 24; 19,$25 ; 19,33 \mathrm{ff}$; 20,$9 ; 20,15$ f.; 20,$16 ; 20,26$ f.; 20,29 f.; 21,7 f.; 21,10 f.; 21,12 ; 21, 18 f.; 22, 3; 22, 26; 22, 33; 22, 34; 24, 4; 24, 29 f.; 25, 4f.; 25, 9; 26, 2. 3. Das gleiche Wort in verschiedener Bedeutung 18, 29;

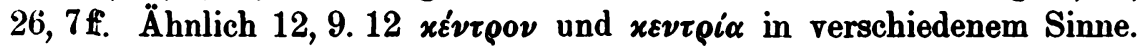

m. Prokatalepsis: 25, $12 \mathrm{ff}$.

Fine abschlieBende Untersuchung der Rhetorik des Akr., die ich, wie ich wiederhole, nicht zu bieten beabsichtige, hätte neben anderen hier beiseite gelassenen Punkten auch sein Verhalten dem Hiatus gegenüber zu prüfen. Hiate, die beispielsweise den von $P$. Wendland ${ }^{1}$ ) bei Philon beobachteten Regeln widersprechen, sind ganze Kapitel hindurch äuBerst selten. ${ }^{2}$ ) Es wäre aber erst durch eine Untersuchung von Wortwahl und Wortstellung zu ergründen, ob dabei Absicht oder Zufall obwaltet. $\mathrm{Zu}$ bemorken ist jedenfalls, daB an zahlroichen Stellen, wie S. 19,$7 ; 19,15 \mathrm{f}$; 19,22 , an welchen eine geringfiigige Umstellung den Hiat verhindert hätte, diese unterblieben ist.

Ich bin auf éine Sphäre, aus welcher Akr. Antikes übernommen hat, die rhetorische, etwas näher eingegangev. Auf eine andere sei wenigstens hingedeutet. An der oben S. 485 ausgeschriebenen Stelle

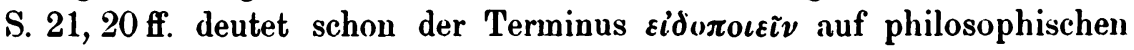

1) Philos Schrift ïber die Vorsehung (Berlin 1892) S. $116 \mathrm{f}$.

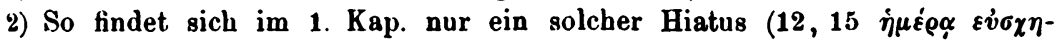
$\mu o ́ v \omega s)$; nach lov 12, 1 ist der Hiatus durch die dort anzunehmende kleine Pause entschuldigt. Für die Kapitel 9-13 sind die entsprechenden Zahlen: $2(18,15$

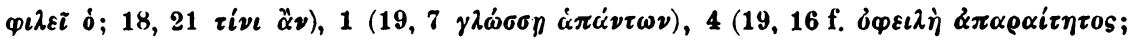

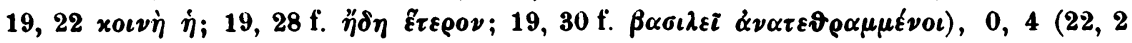

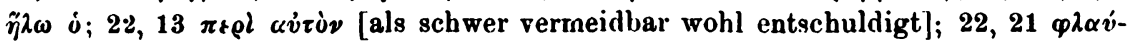

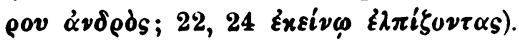

Byzant. Zeitschrift XIV 3 u. 4 . 
EinfluB. Eine nähere Quelle wird sich vielleicht noch nachweisen lassen. Der Gedanke wurzelt in der antiken Philosophie, vgl. Aristot. nikom. Eth. 6,13 S. 1144 b 18; 10, 8 S. 1178 a 16 ff., A. Bonhöffer, Die Ethik des Stoik. Epictet (Stuttgart 1894) S. 214. Bestimmter führt eine andere Stelle auf den Neuplatonismus, mit dem der Verfasser auch sonst Bekanntschaft verrät. ${ }^{1}$ ) S. 12, 9 ff. heiBt es mit Beziehung

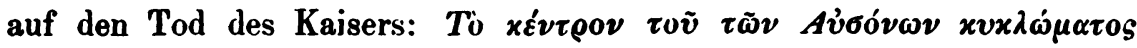

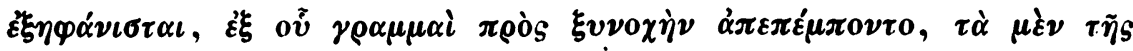

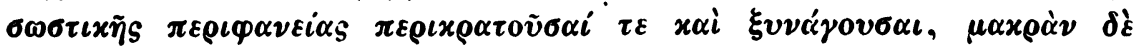

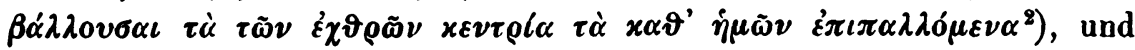

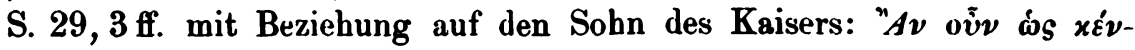

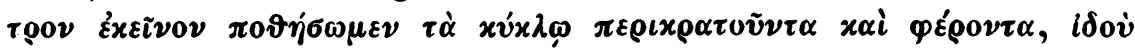

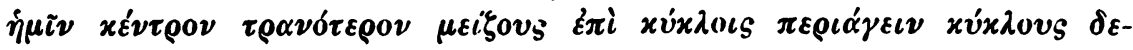

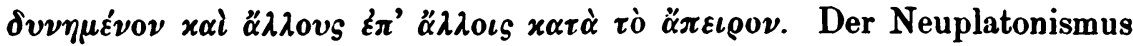
vergleicht das Urwesen mit dem Zentrum eines Kreises, das durch seine Radien alles beherrscht ${ }^{3}$ ), ein Vergleich der, wie dies sehr nahe liegt, bei Plotin 1, 7, 1 a. E. mit dem Bilde der das Weltall durchleuchtenden Sonne in Verbindung gebracht wird. Auch Akr. geht an beiden Stellen vom Zentrumsvergleiche unmittelbar zum Sonnenvergleiche über. Beide Vergleiche sind nun schon vor Akr. dem Königstopos dienstbar gemacht worden, indem sie von dem Beherrscher des Weltalls auf den eines Staates übertragen wurden. So geschieht es an der von mir B. Z. IX (1900) 627 besprochenen Stelle des Maischen Anonymus $\pi$. $\pi 0 \lambda \iota \tau \iota x \tilde{\eta} s \dot{\varepsilon} \pi \iota \tau \eta_{\mu} \mu \eta$ s. Aus dieser oder aus einer ähnlichen Stelle eines andern neuplatonisierenden Fürstenspiegels wird also Akr. die Anregung geschöpft haben.

Die Worte 28, 9 ff. habe ich bereits oben S. 487 auf Herod. 1, 65 zurückgeführt und Parallolon aus Themistios beigebracht. Aber auch hier kommt neben dem rhetorischen EinfluB philosophischer in Frage, und zwar ist es wieder der Neuplatonismus, in dessen Kreise der Verfasser sich bewegt. In seiner Einleitung in die Philosophie hat Elias ${ }^{4}$ )

1) Vgl. B. Z. XIII (1904) $525 \mathrm{f}$.

2) Die $\mathrm{H} 8$ und Heisenberg $\varepsilon \pi \alpha \lambda \lambda \sigma_{\mu \varepsilon \nu \alpha}$ (letzterer verweist im Ind. gramm. u. Verba: Praesens auf das homerische $\varepsilon \pi \hat{\alpha} \lambda \tau o)$.

3) Vgl. die Stellen bei Zeller, Philos. d. Griech. III $2^{4}$ S. 554 Anm. 3. Ebenso

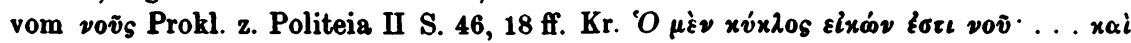

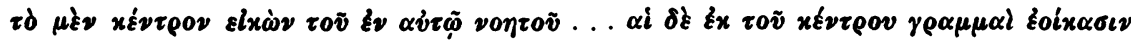

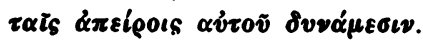

4) J. A. Cramer, Anecd. Gr. Paris. IV (Oxonii 1841) S. 395, 20 ff. Zur Zuteilung an Elias vgl. A. Busse, Comm. in Aristot. Graeca ed. cons. et auct. academ. litt. reg. Boruss. vol. IV pars I (Berlin 1887) S. X. XXXVI ff. [Vgl. jetzt auch David. Proleg. S. 16, 25 ff. - Korrekturnote des Verf.] 
einen Passus, der mit dem des Akr. verglichen diesen Sachverhalt sofort hervortreten läBt.

Elias.

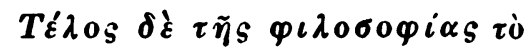

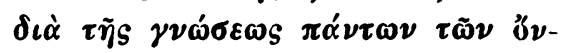

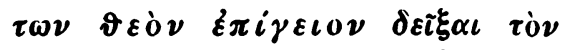

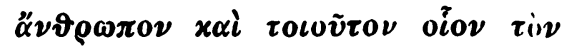

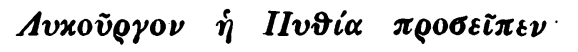

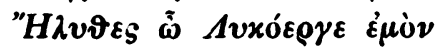
$\pi 0 \pi i \pi i 0 \nu \alpha \nu \eta \dot{\nu}$.

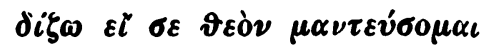
$\grave{\eta} \hat{\varepsilon} x \alpha \grave{\alpha} \ddot{\alpha} \nu \delta \rho \alpha$.
Akropolites.

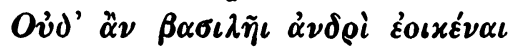

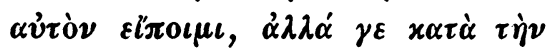

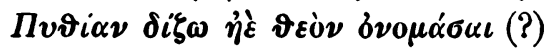

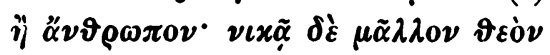

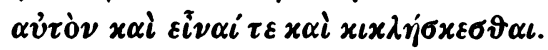

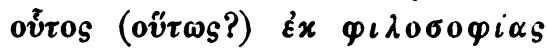

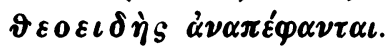

Diese Proben mögen genügen, um zu zeigen, daB hier noch quellenkritische Arbeit zu tun ist, die Erfolg verspricht. Akropolites verdient es sehr wohl, daB man in dieser Weise der Genesis seiner Gedanken nachgehe. So wenig umfangreich sein literarischer NachlaB ist, so spiegelt er doch die byzantinische Geisteskultur des 13. Jahrhunderts wieder. Von dieser werden wir erst dann ein zureichendes Bild erhalten, wenn uns der Werdegang von Männern wie Georgios Akropolites, Nikephoros Blemmydes, Theodoros II Laskaris u. a. durch philologische Analyse ihrer Werke klargelegt ist.

Bern.

Karl Praechter. 